\title{
Actas de la Jornada Técnica sobre "Salud mental de los trabajadores en el tejido empresarial de Castilla y León: estrategias preventivas y tutela reparadora"
}

\author{
celebrada en la Facultad de Derecho de la Universidad de León \\ los días 20 y 21 de septiembre de 2016
}

\section{HENAR ÁLVAREZ CUESTA JAVIER FERNÁNDEZ-COSTALES MUÑIZ}

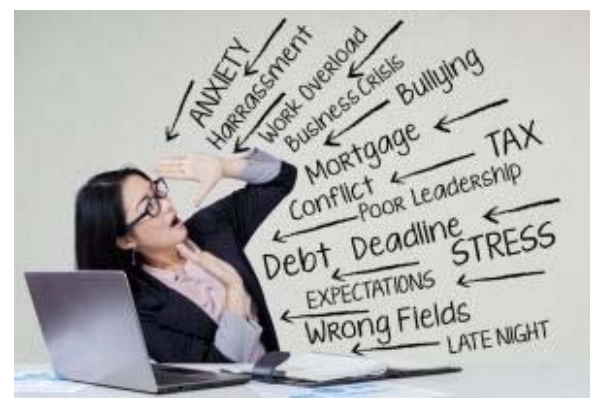

En esta Jornada, en diferentes mesas redondas, participaron miembros del Área de Derecho del Trabajo y de la Seguridad Social de la Facultad de Derecho de la Universidad de León, así como los diferentes actores intervinientes en el ámbito de la salud mental en la Comunidad de Castilla y León, como pueden ser Instituciones Públicas (Dirección General de Trabajo, Instituto de Seguridad e Higiene de Castilla y León, Inspección de Trabajo, Magistratura y Judicatura), interlocutores sociales (sindicatos y asociaciones empresariales), y otras empresas y organismos, como se podrá apreciar en esta relatoría.

Las conclusiones extraídas de dichas mesas redondas se ofrecen a continuación:

La primera de ellas, celebrada el día 20 de septiembre, bajo el título " $\mathrm{La}$ Medicina del Trabajo ante la protección de la salud mental de los trabajadores", contó con los siguientes ponentes:

- Dr. D. José Manuel de la Torre Robles (Doctor en Medicina Preventiva. Hospital de León).

- Dr. D. Serafín de Abajo Olea (Doctor en Medicina del Trabajo. Universidad de León).

- D. Enrique Ortega (Psiquiatra).

- D. Fernando García Paniagua (Director Provincial del INSS).

- Dña. Susana Rodríguez Suárez (Jefa de la Unidad Médica del EVI).

- Profra. Dra. Dña. Consuelo Morán Astorga (Psicóloga. Universidad de León).

Relatores: Juan José Fernández Domínguez y $\mathrm{M}^{\mathrm{a}}$ de los Reyes Martínez Barroso. Profesores de Derecho del Trabajo y de la Seguridad Social de la Universidad de León. 
Interesa destacar de la participación de D. Fernando García Paniagua, Director provincial del INSS de la provincia de León quien aludió a la existencia de un Convenio de colaboración entre el Ministerio de Empleo y Seguridad Social (INSS), y la Comunidad Autónoma de Castilla y León para el control de los días de duración de la Incapacidad Temporal durante el período 2013 a 2016.

A partir de tal convenio se ofrecieron a los asistentes los siguientes datos estadísticos:

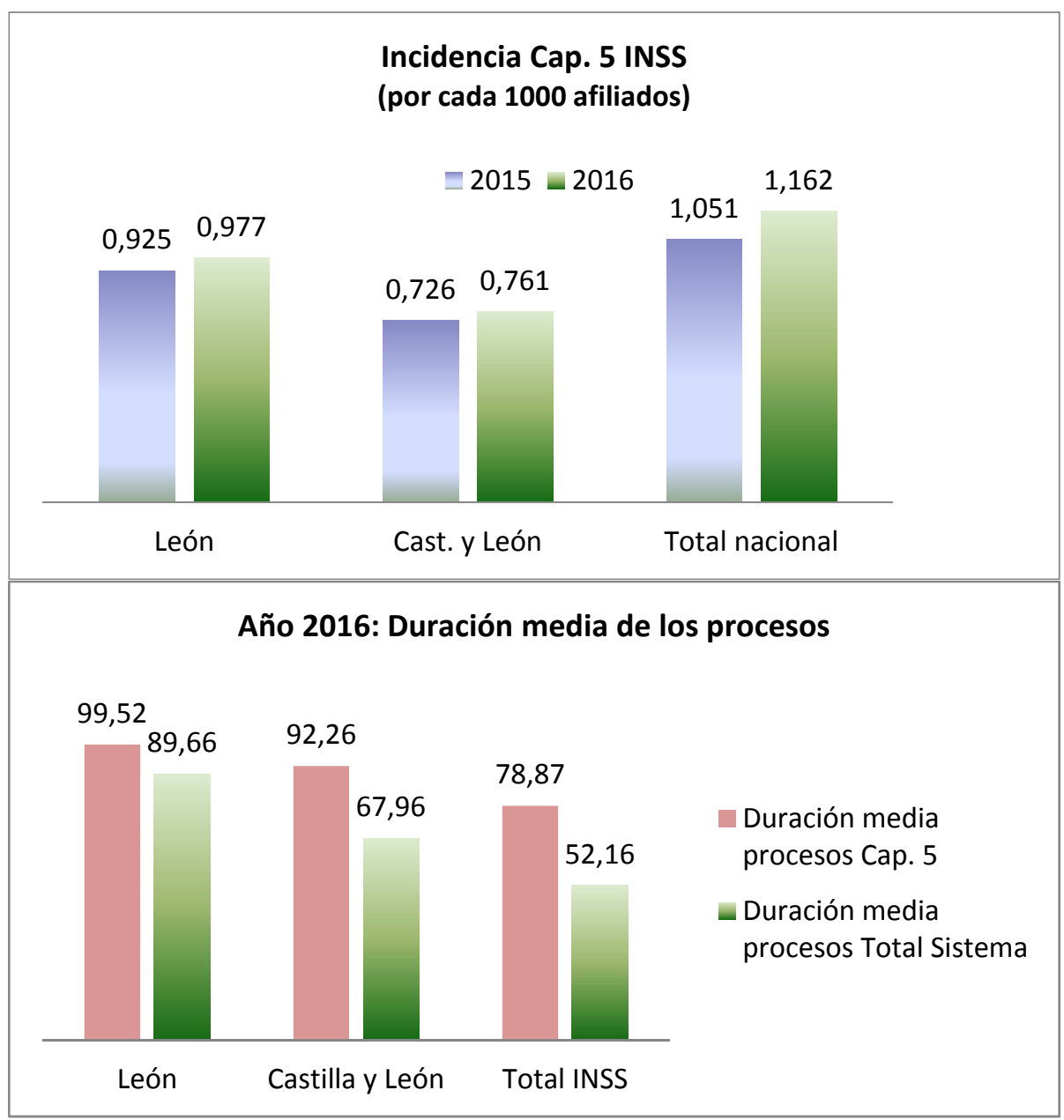

A partir de tales datos es posible llegar a la conclusión de que la incidencia de los procesos de IT causados por patologías del grupo 5 (esto es, las que afectan a la salud mental), es inferior en León, tanto si se compara con el dato a nivel autonómico como nacional. Las consecuencias negativas de un alto índice de prevalencia son:

Incremento de costes salariales a las empresas, lo cual provoca una mayor ineficiencia y una pérdida de competitividad. 
Generación de un clima social favorable al incremento del absentismo y a la dependencia subsidiada, creando entornos sociales caracterizados por la atonía y la falta de competitividad.

Si hubiera que buscar las posibles causas que provocan un peor dato de prevalencia en León respecto a los datos autonómicos y nacionales estas serían las siguientes: a) Población activa envejecida, propia de un entorno socio económico en declive. b) Deficiente prestación del servicio de asistencia sanitaria respecto a otras provincias (listas de espera más dilatadas). c) Deficiente control de los procesos por parte de las inspecciones del SACYL, del INSS y de las Mutuas colaboradoras de la Seguridad Social.

Frente al siguiente diagnóstico, desde la Dirección Provincial del INSS se hacen las siguientes propuestas de mejora:

Mayor comunicación e interacción entre las inspecciones del INSS, del SACYL y de las Mutuas colaboradoras de la Seguridad Social.

Comunicación permanente entre las inspecciones médicas del INSS y los servicios especializados hospitalarios del SACYL en el Área de Salud Mental.

Acceso telemático a todos los historiales médicos de atención especializada del SACYL por parte de la Inspección Médica del INSS.

Fomento de la formación de los inspectores del INSS, SACYL y Mutuas en materia de Salud Mental.

Incremento de la labor protectora en las empresas a través de sus servicios de prevención.

Doña Susana Rodríguez Suárez (Jefa de la Unidad Médica del Equipo de Valoración Médica de Incapacidades) expuso su experiencia profesional poniendo de manifiesto cómo los Inspectores médicos tienen que medir con la mayor objetividad posible, pues una de cada dos personas padece un trastorno emocional a lo largo de su vida (en numerosas ocasiones trastornos adaptativos que van acompañados del consumo de alcohol) que tienen que ver con la edad (normalmente afecta a personas mayores de 50 años), el sector de actividad (inmobiliario, construcción o trabajo autónomo) o el concreto momento económico (la crisis económica ha aumentado las patologías de este tipo, por ejemplo en minería).

Entre un 2 y un $10 \%$ de esos trastornos generan una Incapacidad Permanente. Lo normal es que los trastornos adaptativos generen una IT de 20 o 30 días de duración, mientras que las reacciones de estrés agudas necesitan de unos 7 días para la recuperación y vuelta al trabajo.

También la experiencia muestra que existe una notable relación entre ansiedad y lumbalgia; distimias o alteraciones del humor.

En turno de coloquio el Profesor Mauriz (Profesor Titular de la Universidad de León, acreditado para Catedrático) preguntó a la Mesa si existe alguna correlación entre 
trabajos individualistas o en equipo y la duración de la IT derivada de patologías mentales, considerando la experta que en numerosas ocasiones el trabajo autónomo (destacando el ejemplo de las teleoperadoras) genera una mayor prevalencia de la IT, pues el trabajo en equipo protege frente a ciertas patologías de las aquí estudiadas.

Por último, Doña Consuelo Morán Astorga, Psicóloga y Profesora Titular de la Universidad de León realizó una presentación bajo el título "Estresores del trabajo que pueden afectar a la salud mental", donde, a partir del multidimensional concepto de salud acuñado por la Organización Mundial de la Salud en 1948 (estado de completo bienestar físico, mental y social, y no solamente la ausencia de afecciones o enfermedades) parte de la premisa de que la salud engloba todos los aspectos de la vida, tanto biológicos, como psicológicos.

La salud mental es un concepto positivo que incluye un sentimiento de satisfacción y de plenitud y engloba diversos parámetros (indicadores de eficiencia funcional, de bienestar, de dominio de sí mismo y de competencia personal y ausencia de signos y síntomas psiquiátricos).

A su vez, el trabajo proporciona una serie de elementos que influyen en la salud mental (actividad para utilizar la energía física y mental que tenemos; estructura el tiempo diario, anual...; permite dominar el ambiente: lograr metas, explorar nuevas oportunidades; intercambiar relaciones sociales; estatus, autoestima, sentido de utilidad, etc.). No obstante, existe cierta probabilidad de sufrir algún daño como consecuencia de estar sometido a determinadas condiciones de trabajo.

Entre los diversos estresores que podrían englobarse dentro del concepto de "riesgo laboral" es posible hacer referencia:

a) Estresores del ambiente físico que dificultan e impiden la correcta ejecución del trabajo y que por su peligrosidad pueden provocar en el individuo un sentimiento de amenaza (arquitectura, ruido, iluminación, temperatura, aireación, higiene, vibración,...).

b) Estresores individuales asociados al rol del trabajador (conflicto de rol, ambigüedad de rol, sobrecarga de trabajo - cuantitativa o cualitativa-, infra-carga de trabajo, inseguridad en el trabajo, carrera profesional).

c) Estresores grupales que provienen de la influencia que ejercen los grupos formales y los informales sobre los trabajadores y que derivan de las relaciones personales tanto con superiores, como con subordinados o compañeros (trato desconsiderado o injusto; supervisión muy estrecha; mala supervisión, o ausencia de ella; poca consideración hacia los compañeros, subordinados, jefes; 1 mobbing o acoso laboral).

d) Estresores organizacionales procedentes de las características generales de la organización (políticas de la empresa; sistemas de recompensas económicas; cambios organizacionales).

e) Estresores extra-organizacionales (acontecimientos externos al trabajo: vida familiar, cambios, enfermedades, cambio de residencia, problemas conyugales) que afectan y pueden incrementar el estrés laboral. 
A partir de tales definiciones se expusieron los resultados de un estudio empírico sobre estresores y salud llevado a cabo por la ponente, llegando a la conclusión de que muchos estresores pueden ser perniciosos para el bienestar de los trabajadores pero, los más potentes, son los derivados de las relaciones sociales y del ambiente psíquico y social del entorno laboral.

Dentro de estos estresores grupales aparece el acoso laboral como el más perjudicial para la salud, causando numerosos síntomas físicos y mentales como ansiedad e insomnio y depresión grave.

\section{2}

La segunda mesa redonda, celebrada el día 20 de septiembre con el título " $E l$ papel de la negociación colectiva en la protección de la salud mental de los trabajadores", tuvo la participación de:

- Dña. Covadonga Bernardo (Técnico en Prevención de Riesgos Laborales. Secretaría de Salud Laboral de CCOO).

- D. César Díez González (Asesoría de Salud Laboral de UGT).

- Dña. Cristina García García (Técnico en Prevención de Riesgos Laborales de la Federación Leonesa de Empresarios).

Relatores: Beatriz Agra Viforcos y Javier Fernández-Costales Muñiz. Profesores de Derecho del Trabajo y de la Seguridad Social de la Universidad de León.

Participaron en la mesa redonda Dña. Covadonga Bernardo (técnica de prevención de la Secretaría de Salud Laboral del sindicato CC.OO.), D. César Díaz (experto en Salud Laboral del sindicato UGT) y Dña. Cristina García (técnica de prevención de la FELE), quienes estuvieron acompañados por los relatores Dña. Beatriz Agra y D. Javier Fernández-Costales, ambos profesores titulares de Derecho del Trabajo y de la Seguridad Social en la Universidad de León.

La Ley 31/1995, de 8 de noviembre, de Prevención de Riesgos Laborales (LPRL), proporciona el marco normativo básico para la tutela de la seguridad y salud de los trabajadores. Esta norma exige que en la empresa se identifiquen los riesgos, se eliminen los que puedan ser eliminados, se evalúe el resto y se planifique la actuación a seguir para luchar contra ellos, sin establecer distinciones entre los distintos tipos de riesgo, por lo que procede afirmar que las previsiones en tal sentido alcanzan también a los calificados como psicosociales. La noción enlaza con las cuestiones tratadas en las mesas redondas anteriores, en las que se ha hecho patente la existencia de evidencias científicas en cuya virtud existen ciertas características del trabajo (el exceso de exigencia, la falta de influencia y de desarrollo, la escasez de compensaciones, la dificultad de afrontar la doble jornada de trabajo...), fallos en su organización y deficientes relaciones interpersonales en el seno de la empresa con capacidad para afectar negativamente a la salud del trabajador.

Sea como fuere, la LPRL es una ley de mínimos que únicamente proporciona un marco que debe ser completado, ya sea vía reglamentaria (como efectivamente ha 
ocurrido), ya mediante la negociación colectiva. El complemento de los interlocutores sociales resulta especialmente importante en relación con el riesgo psicosocial, pues, a diferencia de lo que ocurre con otros (biológicos, ruido, vibraciones...), no existe desarrollo infralegal específicamente aplicable al mismo.

Esta labor de los interlocutores sociales se puede y debe desarrollar en distintos niveles (en los comités de seguridad y salud, en los acuerdos de empresa, en los convenios marco y sobre materias concretas...), destacando entre todos ellos el convenio colectivo, fundamentalmente por dos motivos: de un lado, porque aglutina la regulación aplicable a las distintas condiciones de trabajo, por lo que constituye ámbito idóneo para la inclusión transversal de consideraciones preventivas (integración de la prevención en la empresa que viene exigida por la propia LPRL); de otro, porque el convenio colectivo cuenta con un potencial inmenso en lo relativo al riesgo psicosocial dada su esencial adaptabilidad a la realidad del concreto sector/empresa, así como a su carácter temporal, con la consiguiente capacidad para acomodarse a los cambios con cierta rapidez.

Teniendo esto presente, la norma pactada es un lugar óptimo para establecer una regulación de la organización del trabajo amigable para el trabajador, para orientar sobre los factores de riesgo más importantes del sector/empresa, para determinar el método de valoración de los riesgos psicosociales, para proporcionar una regulación de la vigilancia de la salud específicamente relacionada con las condiciones de trabajo del sector/empresa,...

Siendo este el deseo, procede interrogarse sobre cuál es la realidad. Desde el punto de vista sindical, la respuesta no resulta en modo alguno optimista, en tanto todavía son muchos los convenios que no incorporan cláusulas relativas a la salud laboral (y muchísimos más los que obvian por completo lo relativo a los riesgos psicosociales), que se limitan a remitir a la ley o reproducir su tenor (lo que, más allá del valor didáctico o pedagógico, poco aporta) o que se conforman con incorporar genéricas y vacías declaraciones de intenciones. En definitiva, y salvo honrosas excepciones debidamente puestas de manifiesto a lo largo de la sesión (donde se destacó la negociación colectiva de la industria química), el tema permanece todavía al margen del convenio, con la salvedad de las crecientes previsiones relacionadas con algunos aspectos concretos; señaladamente, el acoso en sus diversas variables (sexual, moral, discriminatorio), aunque con un enfoque claramente reactivo (disciplinario) y apenas proactivo (prevención, formación, sensibilización, información...).

¿Cuál puede ser la causa de este silencio o de este tratamiento insuficiente? El desarrollo de la mesa redonda puso sobre la mesa algunas cuestionas dignas de mención:

Primero, la generalizada ausencia de cultura preventiva en la empresa española (tanto entre empresarios como entre trabajadores), donde las obligaciones preventivas se cumplen más de manera formal y burocrática que sustantiva y real, algo predicable de todo el conjunto de la seguridad y salud laboral, en general, y de los aspectos psicosociales, en particular, en tanto en el orden de importancia otorgada a los distintos riesgos en la empresa, estos ocupan, sin duda, el último lugar. 
Segundo, la falta de interés por parte de los trabajadores, que, intranquilos por cuestiones que consideran más importantes, sólo se preocupan de la salud cuando la pierden; a ello se añade el hecho de que la mayor parte de los empresarios continúan viendo la prevención de riesgos laborales como un gasto y no como una inversión. Una vez más, se trata de planteamientos que afectan a cualesquiera riesgos, pero que pesan en superior medida sobre aquellos que parecen considerarse de segundo orden, los psicosociales.

Tercero, y en relación con lo anterior, los efectos de la crisis económica, que ha significado un fuerte golpe para los avances que se estaban realizando en las empresas, sobre todo en aquellas de cierto tamaño y con presencia de representantes del personal. Ante la necesidad acuciante de atender a otros temas supuestamente más urgentes (despidos, salarios...), la importancia otorgada a una materia todavía incipiente ha decrecido exponencialmente.

Cuarto, la propia dificultad de ocuparse a través del convenio colectivo de una materia cuyo tratamiento resulta muy complejo y poliédrico, tal y como han reconocido los profesionales médicos, psicólogos y/o técnicos de prevención que han participado en estas Jornadas.

Quinto, en el concreto caso de Castilla y León es muy importante tener presente la naturaleza de su tejido empresarial. Hay un claro predominio de la PYME, la temporalidad en la contratación es elevada, la externalización de la prevención es la opción mayoritaria... factores todos ellos que dificultan el cumplimiento efectivo y eficiente de las exigencias establecidas por la LPRL y su normativa de desarrollo y complemento.

Frente a esta realidad, resulta esencial abordar el tema en toda su amplitud y atendiendo a sus múltiples facetas, sobre todo en cuanto hace al planteamiento de cambios en la organización del trabajo, actuando sobre el origen mediante acciones que, adaptadas a la realidad de cada entidad productiva, combinen la protección de los trabajadores con la atención a las necesidades productivas de la empresa. En este cambio deben participar todos los implicados, incluidos los sindicatos, que han de asumir la salud laboral como un elemento esencial de la acción sindical, luchando porque, pese a las dificultades, las mejoras que se vayan logrando en la esfera de la seguridad y salud en el trabajo también alcancen a los aspectos psicosociales. La negociación colectiva se alza como esencial en este empeño.

En todo caso, la negociación colectiva del futuro no debería conformarse con los estrechos planteamientos seguidos hasta la fecha. Incluso en aquellos convenios donde se ha incluido alguna previsión de interés referida a la salud laboral, no se ha planteado esta como algo integral y transversal, sino que se ha confinado a apartados específicos. Esta forma de actuar no es válida para el tratamiento de los riesgos laborales, y menos aun cuando se trata de los psicosociales, indisolublemente unidos a una organización del trabajo y conectados con prácticamente la totalidad de condiciones de trabajo: salario, jornada, horario, conciliación, sistema de clasificación, promoción, formación... 
Sin embargo, y conforme ya se adelantó, en aquellos convenios que sí contemplan la prevención de riesgos laborales, lo hacen mediante un tratamiento puntual y poco integrador, con previsiones marginales ajenas a la dinámica cotidiana del proceso productivo y de la vida de la empresa: protocolos a aplicar en hipótesis de acoso u otras formas de violencia, disposiciones relacionadas a la vigilancia de la salud que sólo en unos pocos casos aluden a la salud psíquica... y poco más. Parece que el mayor obstáculo para asumir el deseable planteamiento integrador (reclamado por el legislador, recuérdese) serán las dificultades técnicas, que podrían ser salvadas mediante el auxilio de profesionales cualificados para orientar sobre cómo conseguir que la regulación incorporada al convenio incluya la consideración de los efectos de su puesta en práctica sobre la salud mental de los trabajadores.

Dado que entre el momento actual y la realización de la meta apuntada media una enorme distancia, entre tanto parece aconsejable avanzar hacia una etapa intermedia y provisional. En efecto, a la espera de alcanzar tan ambicioso objetivo (que parece alzarse como mero desideratum o ideal), habrá que intentar lograr, al menos, que a la prevención de riesgos psicosociales se le otorgue el valor que merece, para lo cual parece opción óptima servirse de acuerdos específicamente destinados a ellos a fin de que la materia no permanezca como cuestión de segundo orden dentro del convenio. Entre tanto, los mayores logros parecen seguir en el seno de la responsabilidad social corporativa, bajo la forma de planes, protocolos... cuyo valor es innegable, pero que, dado su componente de práctica unilateralidad, se ven privados de los beneficios y garantías que reportaría el empleo de instrumentos consensuados y de aplicación imperativa.

La tercera mesa, celebrada el día 21 de septiembre con el título "El papel de

3 las Administraciones Públicas en la protección de la salud mental de los trabajadores: intervenciones preventivas y reparadoras", tuvo como ponentes a:

- Dña. Ana Guerrero Folgueras (Jefa de la Oficina Territorial de Trabajo de León).

- D. José Ámez del Pozo (Técnico de Prevención de la Universidad De León).

- D. Fernando Galindo Meño (Jefe de la Inspección de Trabajo de León).

- Ilmo. D. Jaime de Lamo Rubio (Magistrado del Juzgado de lo Social núm. 1 de León).

- D. José Miguel Muñoz Bellido (Director del Centro de Seguridad y Salud Laboral de Castilla y León).

- Teniente Coronel (EA) Félix Santos Álvarez (Jefe del Órgano de Dirección y Secretario del Centro. Academia Básica del Aire. Aeródromo Militar de la Virgen del Camino. León).

Relatores: Susana Rodríguez Escanciano y Roberto Fernández Fernández. Profesores de Derecho del Trabajo y de la Seguridad Social de la Universidad de León.

El papel preponderante de las Administraciones Públicas en la prevención de cualquier tipo de riesgo derivado de la actividad laboral está fuera de toda duda, tanto 
en su actuación como poder público como en su condición de empleador. Dicha importancia adquiere especial significado cuando de riesgos psicosociales se trata, no en vano su carácter silente u oculto bajo otras dolencias físicas o psíquicas junto con la propia organización sumamente jerarquizada en las Administraciones, hacen de ellas uno de los espacios donde con mayor frecuencia acaecen estos riesgos de atender a la información estadística existente.

De esta manera, y por un lado, las Administraciones Públicas son las encargadas de diseñar las políticas preventivas necesarias para luchar contra los riesgos psicosociales, diseñando las herramientas legislativas, normativas y planificadoras adecuadas que luego puedan ser utilizadas por los agentes implicados en los distintos ámbitos de la producción; en esta tarea, y por solo citar algunos ejemplos, están capacitadas para elaborar protocolos de actuación, mapas de riesgos, campañas de sensibilización, asesoramiento y un largo etcétera de todos conocido. Junto a esta labor proactiva, no cabe obviar el papel reactivo que tiene encomendado la Administración a través del cuerpo de Inspectores y Subinspectores de trabajo, quienes tienen la fundamental de fiscalizar el cumplimiento de la normativa preventiva $\mathrm{y}$, en caso de que constaten alguna contravención, proponer las sanciones correspondientes a la autoridad laboral. Todo ello sin olvidar la intervención de los órganos judiciales del orden social para resolver los conflictos de intereses contrapuestos entre los sujetos del contrato.

Por otro, en su condición de empresario debe implementar en sus centros de trabajo cuantas medidas resulten necesarias para prevenir los daños a la salud de sus empleados, bien sean funcionarios, bien sean contratados laborales, atendiendo a las condiciones en las que se desarrolla la actividad laboral, habida cuenta de la heterogeneidad de ámbitos funcionales y territoriales en los que dispensa servicios públicos la Administración.

Sobre las premisas de partida expuestas, se ha considerado necesario contar con la presencia de especialistas en la materia que aporten distintos puntos de vista tanto sobre los problemas que se encuentran en su actividad profesional diaria, como sobre las buenas prácticas diseñadas e implantadas para minimizar los efectos de los riesgos en la salud mental de los empleados públicos. De esta manera, cabe agrupar las conclusiones alcanzadas en la Mesa Redonda en las siguientes fundamentales:

$1^{\mathrm{a}}$.- Es menester destacar la trascendencia que la participación y colaboración de los trabajadores presenta para detectar y luchar problemas de salud mental en los puestos de trabajo públicos. La práctica demuestra que se tiene trabajar con muestras muy pequeñas y en muchos casos poco significativas de las que hay que extraer conclusiones generales, ante la escasa participación de los empleados públicos. Esta circunstancia puede explicarse por la escasa motivación a la hora de contestar cuestionarios sobre riesgos psicosociales y la falsa creencia de la poca efectividad de las posibles medidas a adoptar cuando se denuncia y detecta un caso real.

$2^{\mathrm{a}}$.- Para intentar superar los obstáculos anteriores, deben adquirir un protagonismo destacada las campañas de sensibilización y los estudios destinados a realizar mapas de riesgos, sobre todo en aquellas actividades donde la experiencia demuestra que los 
trabajadores pueden ser más proclives a desarrollar problemas de salud mental, tal y como sucede en paradigmático ejemplo con los call-centers y actividades análogas, en los cuales el contacto telefónico continuo con otras personas y la presión ejercida para vender sus productos en una época de crisis como la actual, hace que el cumplimiento de los objetivos marcados someta a las trabajadores a una presión y niveles de estrés difícilmente soportables.

$3^{\text {a }}$ - A pesar de la frecuencia del riesgo, no es posible obviar, como señalan las estadísticas, que hay un escaso nivel de denuncias sobre acosos y otros comportamientos análogos, siendo muchos los casos que quedan ocultos o no se puede demostrar las conductas presuntamente ilícitas. En este sentido, aquellas Administraciones encargadas de fiscalizar y sancionar las mismas, no solamente deben poner en el foco en dicha tarea, sino también avanzar en su labor de asesoramiento y apoyo a las empresas y trabajadores afectados, de manera que formando mejor a todos los implicados en el proceso productivo, más fácil será visibilizar los factores de peligro.

4. - Tampoco la vía judicial es demasiado utilizada, y cuando se hace, en la mayor parte de las ocasiones el pleito versa sobre la calificación como profesional o común de la contingencia, la extinción causal del contrato a instancias del trabajador o sobre la vulneración de algún derecho fundamental y la cuantificación de la indemnización anudada a la posible contravención. En cualquier caso, los mayores problemas se plantean a la hora de probar los hechos ilícitos y que la conducta realizada es realmente hostigadora y no se trata de un simple conflicto entre las partes sin ningún ánimo nocendi y atentatorio contra la dignidad.

$5^{\text {a }}$.- En ciertos ámbitos del empleo público, los riesgos psicosociales adquieren un cariz particular, tal y como sucede en las fuerzas armadas donde los empleados, en muchas ocasiones, tienen que desempeñar su actividad en condiciones especialmente peligrosas y sometidos a un gran estrés, al tener que tomar decisiones de vital importancia en pocos segundos. Las vivencias en tales destinos marcan para siempre y pro futuro su personalidad, necesitando al regreso terapias que les permitan superar los miedos y ansiedades adquiridas en las misiones en zonas conflictivas.

\section{4}

La cuarta mesa redonda, celebrada el día 21 de septiembre, bajo el título " $\mathrm{La}$ protección de la salud mental de los trabajadores por parte de los servicios de prevención ajenos”, contó con los siguientes intervinientes:

- D. David Ramos (Técnico de Prevención del Servicio de Prevención de PREVILEY).

- D. Gonzalo Mañes (Formador en Prevención de Riesgos Laborales).

- D. Joaquín Vives (Técnico de Prevención del Servicio de Prevención de ASPY).

- D. José Antonio Pérez de la Fuente Cortina (Coordinador Ergonomía y Psicosociología DR Cantábrica. Premap).

- D. José Antonio Campelo (Técnico de Prevención del Grupo Alsa).

- D. Alejandro Cuervo (Auditor de Prevención). 
Relatores: Henar Álvarez Cuesta, Rodrigo Tascón López y José Gustavo Quirós Hidalgo. Profesores de Derecho del Trabajo y de la Seguridad Social de la Universidad de León.

D. Gonzalo Mañes, formador de Prevención de Riesgos Laborales, destacó la conveniencia y necesidad de que los riesgos sicosociales fueran objeto de la debida formación en materia de prevención de riesgos laborales tanto a los trabajadores como a sus representantes. No obstante, reconoció el déficit de formación que existe en la práctica en la gran mayoría de empresas de Castilla y León, sobre todo en las pequeñas y medianas, las más numerosas, y además no solo sobre este aspecto, sino en general, consistiendo en muchos casos la formación en materia preventiva en la aportación al trabajador de un dossier documental sin la eficacia real que la prevención requiere. A tal efecto, expuso una experiencia formativa en la que participó activamente, desarrollada en forma de sesiones que, lejos de responder a una simple exposición teórica, consistió en la presentación activa y en un profundo debate e intercambio de opiniones con los trabajadores sobre supuestos reales de materialización de riesgos psicosociales en el trabajo, para tratar de poner de manifiesto y en sus justos términos el alcance real, muchas veces desconocido, de los mismos.

D. Joaquín Vives, Director de la Delegación de León de ASPY Prevención y D. José Antonio Campelo, Coordinador de Ergonomía y Psicosociología DR Cantábrica Premap, expusieron la forma en que sus respectivos Servicios de Prevención afrontan en la práctica y en el día a día de las empresas para las que prestan servicios el tratamiento de los riesgos psicosociales. Pusieron de manifiesto el desconocimiento que las empresas, a salvo de las de mayor tamaño, tienen de la necesidad, o al menos conveniencia, de incluir este tipo de riesgos en sus planes de prevención y la reticencia que muestran para ello. No obstante, señalaron que en su labor preventiva es cada vez más habitual que, a pesar de dichos obstáculos, la gestión de la prevención de riesgos en las empresas incluyan la previsión, eliminación o planificación de este tipo de riesgos. Es más, también recalcaron que en sus respectivas entidades preventivas, existe un departamento específico organizado jerárquica y verticalmente, más o menos numeroso, y en su mayor parte conformado por psicólogos, que tiene como cometido apoyar individualmente la planificación y tratamiento de estos riesgos en cada caso concreto y, a mayores, diseñar de forma general la actuación en la materia del Servicio de Prevención.

D. José Antonio Campelo, Técnico de Prevención del Grupo ALSA, expuso la experiencia de las empresas del grupo, dedicadas al transporte de viajeros, en el tema de los riesgos sicosociales. En este sentido, relató que, a partir de la directa interlocución con los trabajadores y sus representantes, se pudieron poner de manifiesto los principales riesgos sicosociales, consistentes, entre otros, en el estrés por la necesidad de cumplir los horarios marcados por las administraciones cuyas concesiones explotan y, especialmente, el estrés y las agresiones sufridas por los usuarios de los servicios. A tal efecto, expuso igualmente las medidas adoptadas, muchas de ellas a propuesta de los propios trabajadores, y la relativa eficacia de las mismas. Por tal motivo, resaltó la necesidad de una actualización constante de la planificación de los riesgos laborales, y entre ellos de los psicosociales. 
En último lugar, intervino D. Alejandro Cuervo, Auditor de Prevención, para reiterar lo ya afirmado por anteriores ponentes sobre la resistencia del empresariado de menor tamaño a incluir el tratamiento de los riesgos psicosociales en la prevención, con el dato adicional de la inexistencia de obligación de someter a auditoría la gestión de riesgos laborales que en muchos de los casos son gestionadas directamente por el empresario. Adicionalmente, señaló que, por el contrario, en las de mayor entidad es cada vez más habitual que se aborde su evaluación y planificación mediante alguno de los procedimientos existentes al efecto, siendo el del INSHT el más habitual y en la gran mayoría de los casos a través de cuestionarios a los trabajadores. A continuación, expuso también su experiencia comparando el sistema de prevención y auditoría de riesgos psicosociales en otros países del entorno europeo y culminó precisando que, a su juicio, el tema resulta muy complejo porque en muchos casos se está dando el tratamiento de riesgos a lo que en realidad son resultados ya manifestados.

En general, todos los ponentes coincidieron, al igual que en mesas anteriores, en destacar la dificultad para llevar a cabo un tratamiento y una valoración general a los riesgos psicosociales, pues más allá de los que derivan de circunstancias puramente laborales y de la interacción y de la relación social con otras personas de la empresa, en muchos casos atienden en su origen, manifestación y consecuencias a condiciones puramente personales.

La ponencia final de cierre, titulada "Salud mental de los trabajadores:

5 tutela reparadora”, corrió a cargo del Prof. Dr. D. Jesús Lahera Forteza, Profesor Titular de Derecho del Trabajo y de la Seguridad Social. Universidad Complutense de Madrid. 